\title{
ON ZEROS OF A SYSTEM OF POLYNOMIALS AND APPLICATION TO SOJOURN TIME DISTRIBUTIONS OF BIRTH-AND-DEATH PROCESSES
}

\author{
KEN-ITI SATO
}

ABSTRACT. Zeros of the following system of polynomials are considered:

$$
\left\{\begin{array}{l}
P_{0}(x)=1, \\
P_{1}(x)=B_{0}-A_{0} x, \\
P_{n+1}(x)=\left(B_{n}-A_{n} x\right) P_{n}(x)-C_{n} P_{n-1}(x) \text { for } n \geq 1 .
\end{array}\right.
$$

Numbers of positive and negative zeros are determined and a separation property of the zeros of $P_{m}(x)$ and $P_{n}(x)$ is proved under the condition that $C_{n}>0$ and $P_{n}(0)>0$ for every $n$. No condition is imposed on $A_{n}$. These results are applied to determination of the distribution of a sojourn time with general (not necessarily positive) weight function for a birth-and-death process up to a first passage time. Unimodality and infinite divisibility of the distribution follow.

1. Introduction. In moment problems, in theory of orthogonal functions, and in probability theory, the following system of polynomials appears:

$$
\left\{\begin{array}{l}
P_{0}(x)=1 \\
P_{1}(x)=B_{0}-A_{0} x \\
P_{n+1}(x)=\left(B_{n}-A_{n} x\right) P_{n}(x)-C_{n} P_{n-1}(x) \text { for } n \geq 1
\end{array}\right.
$$

where $A_{n}, B_{n}$, and $C_{n}$ are real and $A_{n}>0, C_{n}>0$. Important features of these polynomials are that $P_{n}(x)$ is of degree $n$ and has $n$ simple real zeros, and that the zeros of $P_{n+1}(x)$ separate the zeros of $P_{n}(x)$. Under an additional condition Yamazato [16] proves that, if $m<n$, then between any consecutive zeros of $P_{m}(x)$ there exists at least one zero of $P_{n}(x)$. His additional condition is equivalent to that $P_{n}(0)$ is positive for every $n$. If normalization $P_{n}(0)=1$ is made, then $B_{0}=1$ and $B_{n}=1+C_{n}$ for $n \geq 1$. This result is rediscovery of Stieltjes's result, as is explained in [16]. Using this fact, Yamazato makes refinement of Keilson's result [5] on distributions of first passage times for birth-and-death processes and, further, exactly determines the class of these distributions. The purpose of the present

Received by the editors July 9, 1987.

1980 Mathematics Subject Classification (1985 Revision). Primary 26C10; Secondary 60E99, $60 \mathrm{~J} 27$.

Key words and phrases. Zeros of polynomials, sojourn time distribution, birth-and-death process, unimodal, infinitely divisible.

The research was partly done while the author was visiting the Institute for Mathematics and its Applications, University of Minnesota, in November 1985. This paper was announced in [9] under the title Extension of Yamazato's results on zeros of a system of polynomials and application to sojourn time distributions. 
paper is, first, to extend the properties of zeros of the system of polynomials to a case where the coefficients $A_{n}$ are not necessarily positive and, second, to apply the extension to finding distributions of sojourn times with weight not necessarily positive.

In $\S 2$ we will formulate our main results. Some classes generated by exponential distributions are introduced in $\S 3$ and our results on distributions of sojourn times for birth-and-death processes are given in $\S 4$. Proof of the theorems in $\S 2$ is given in $\S 5$ and, finally, the results in $\S 4$ are established in $\S 6$.

2. Main results. Consider the system of polynomials defined by (1.1). Assume the following:

$$
\begin{gathered}
C_{n}>0 \quad \text { for } n \geq 1, \\
P_{n}(0)>0 \quad \text { for } n \geq 1 .
\end{gathered}
$$

Note that these assumptions imply $B_{n}>0$ for $n \geq 0$. No condition is imposed on the constants $A_{n}$. They can be arbitrary real numbers. Denote by $N^{+}(n)$ [resp. $N^{-}(n)$ ] the number of $k$ 's such that $0 \leq k \leq n-1$ and $A_{k}>0$ [resp. $A_{k}<0$ ]. Let $N(n)=N^{+}(n)+N^{-}(n)$. An open interval with endpoints $a, b$ is denoted by $(a, b)$. We mean by the highest coefficient of $P_{n}(x)$ the coefficient of the term with the highest power in $P_{n}(x)$.

THEOREM 2.1. (i) The degree of $P_{n}(x)$ is $N(n)$. The sign of the highest coefficient of $P_{n}(x)$ is $(-1)^{N^{+}(n)}$.

(ii) The zeros of $P_{n}(x)$ are simple and real. The number of the positive zeros of $P_{n}(x)$ is $N^{+}(n)$ and that of the negative zeros of $P_{n}(x)$ is $N^{-}(n)$.

THEOREM 2.2. (i) Designate the $N(n)+1$ points consisting of the zeros of $P_{n}(x)$ and the point 0 as $a_{1}^{n}<a_{2}^{n}<\cdots<a_{N(n)+1}^{n}$. Let $0 \leq m<n$. Then, for each $j(1 \leq j \leq N(m))$, the interval $\left(a_{j}^{m}, a_{j+1}^{m}\right)$ contains at least one zero of $P_{n}(x)$. Moreover, the interval $\left(a_{N(m)+1}^{m},+\infty\right)$ contains at least one zero of $P_{n}(x)$ if $A_{m}>0$; the interval $\left(-\infty, a_{1}^{m}\right)$ contains at least one zero of $P_{n}(x)$ if $A_{m}<0$.

(ii) Let $0 \leq m<n$. The number of common positive [resp. negative] zeros of $P_{m}(x)$ and $P_{n}(x)$ is at most $\min \left\{N^{+}(m), N^{+}(n)-N^{+}(m+1)\right\}\left[\right.$ resp. $\min \left\{N^{-}(m)\right.$, $\left.\left.N^{-}(n)-N^{-}(m+1)\right\}\right]$

Using the notation in Theorem 2.2(i), we get the following theorem as a special case $n=m+1$.

THEOREM 2.3. The $N(m+1)$ simple zeros of $P_{m+1}(x)$ are located as follows. If $A_{m}>0$, then each of the intervals $\left(a_{1}^{m}, a_{2}^{m}\right), \ldots,\left(a_{N(m)}^{m}, a_{N(m)+1}^{m}\right),\left(a_{N(m)+1}^{m},+\infty\right)$ contains exactly one zero of $P_{m+1}(x)$. If $A_{m}<0$, then each of the intervals $\left(-\infty, a_{1}^{m}\right),\left(a_{1}^{m}, a_{2}^{m}\right), \ldots,\left(a_{N(m)}^{m}, a_{N(m)+1}^{m}\right)$ contains exactly one. If $A_{m}=0$, then each of $\left(a_{1}^{m}, a_{2}^{m}\right), \ldots,\left(a_{N(m)}^{m}, a_{N(m)+1}^{m}\right)$ contains exactly one.

3. Classes generated by exponential distributions. Let us introduce some classes of distributions generated by exponential distributions on $\mathbf{R}_{+}=[0,+\infty)$ and $\mathbf{R}_{-}=(-\infty, 0]$. For $a>0$ [resp. $a<0$ ], let $\mu_{a}$ denote the exponential distribution on $\mathbf{R}_{+}\left[\right.$resp. $\left.\mathbf{R}_{-}\right]$with mean $1 / a$, that is, $\mu_{a}$ is supported on $\mathbf{R}_{+}\left[\right.$resp. $\left.\mathbf{R}_{-}\right]$and $\mu_{a}(d x)=|a| e^{-a x} d x$ on $\mathbf{R}_{+}\left[\right.$resp. $\left.\mathbf{R}_{-}\right]$. Define, for any positive integer $k$, classes 
$M E_{++}^{k}, M E_{+0}^{k}, M E_{+}^{k}, C E_{+}^{k}$ and $M E_{--}^{k}, M E_{-0}^{k}, M E_{-}^{k}, C E_{-}^{k}$ as follows. The class $M E_{++}^{k}$ [resp. $M E_{--}^{k}$ ] is the set of mixtures of $k$ distinct exponential distributions on $\mathbf{R}_{+}$[resp. $\mathbf{R}_{-}$]. That is, $\mu \in M E_{++}^{k}$ [resp. $M E_{--}^{k}$ ] iff

$$
\mu=\sum_{i=1}^{k} p_{i} \mu_{a_{i}}, \quad p_{i}>0 \quad(1 \leq i \leq k), \quad \sum_{i=1}^{k} p_{i}=1,
$$

and $a_{1}, \ldots, a_{k}$ are distinct positive [resp. negative] numbers. The class $M E_{+0}^{k}$ [resp. $M E_{-0}^{k}$ ] is the set of mixtures of the delta distribution $\delta_{0}$ at 0 and $k$ distinct exponential distributions on $\mathbf{R}_{+}$[resp. $\mathbf{R}_{-}$]. Namely, $\mu \in M E_{+0}^{k}$ [resp. $M E_{-0}^{k}$ ] iff

$$
\mu=p_{0} \delta_{0}+\sum_{i=1}^{k} p_{i} \mu_{a_{i}}, \quad p_{i}>0(0 \leq i \leq k), \quad \sum_{i=0}^{k} p_{i}=1,
$$

and $a_{1}, \ldots, a_{k}$ are distinct positive [resp. negative] numbers. We define

$$
M E_{+}^{k}=M E_{++}^{k} \cup M E_{+0}^{k}, \quad M E_{-}^{k}=M E_{--}^{k} \cup M E_{-0}^{k} .
$$

The class $C E_{+}^{k}$ [resp. $C E_{-}^{k}$ ] is the set of convolutions of $k$ (not necessarily distinct) exponential distributions on $\mathbf{R}_{+}$[resp. $\mathbf{R}_{-}$], that is, $\mu \in C E_{+}^{k}$ [resp. $C E_{-}^{k}$ ] iff $\mu=\mu_{a_{1}} * \cdots * \mu_{a_{k}}$, where $a_{1}, \ldots, a_{k}$ are positive [resp. negative] numbers. Let $M E_{+0}^{0}, M E_{+}^{0}, C E_{+}^{0}, M E_{-0}^{0}, M E_{-}^{0}$, and $C E_{-}^{0}$ denote the set consisting of a single element $\delta_{0}$. For any two classes $A^{(1)}, A^{(2)}$ of distributions on $\mathbf{R}=(-\infty,+\infty)$, denote by $A^{(1)} * A^{(2)}$ the class of $\mu$ such that $\mu=\mu^{(1)} * \mu^{(2)}, \mu^{(1)} \in A^{(1)}$, and $\mu^{(2)} \in A^{(2)}$; we call $\mu^{(1)} * \mu^{(2)}$ an expression of $\mu$ in $A^{(1)} * A^{(2)}$. We define

$$
E(k, l, r, s)=M E_{+}^{k} * C E_{+}^{l} * M E_{-}^{r} * C E_{-}^{s}
$$

for nonnegative integers $k, l, r, s$.

Notice that infinite divisibility and unimodality are known for distributions of the above classes. It is well known that exponential distributions on $\mathbf{R}_{+}$and $\mathbf{R}_{-}$are infinitely divisible and that the class of infinitely divisible distributions is closed under convolution. C. Goldie [1] proves that mixtures of exponential distributions on $\mathbf{R}_{+}$are infinitely divisible (see also F. Steutel [13]). Thus all distributions in $E(k, l, r, s)$ are infinitely divisible. Distributions in $M E_{+}^{k}$ or $M E_{-}^{l}$ are obviously unimodal with mode 0 . Hence distributions in $M E_{+}^{k} * M E_{-}^{l}$ are unimodal with mode 0 (see Lemma 6.1 of Sato-Yamazato [12] or Lemma 3 of Sato [11]). Exponential distributions are strongly unimodal; namely, for any unimodal distribution $\nu$, the convolution $\nu * \mu_{a}$ is unimodal (see Ibragimov [2]). It follows that all distributions in $E(k, l, r, s)$ are unimodal.

A distribution $\mu$ is called strictly unimodal if there is a point $a$ (called the mode of $\mu$ ) such that $\mu$ has density on $(-\infty, a)$ and $(a,+\infty)$ and the density is increasing on $(b, a)$ and decreasing on $(a, c)$, where $b$ and $c$ are the left and right extremities, respectively, of the support of $\mu$. (We use in this paper the words "increase" and "decrease" in the strict sense.) Sato [11] shows that any distribution in $E(k, l, r, s)$ is strictly unimodal and has real-analytic densities on $(-\infty, 0)$ and $(0,+\infty)$, and gives explicit bounds for its mode. Other bounds for modes are given in Sato [10] for general unimodal distributions in terms of absolute moments or central absolute moments. 
Let $\mu$ be a distribution on $\mathbf{R}$. Define $\hat{\mu}(x)$ by

$$
\hat{\mu}(x)=\int e^{x y} \mu(d y)
$$

for real $x$ such that the right-hand side is finite. If it is finite for $x$ on an interval $\left(c_{1}, c_{2}\right)$, then the same integral extends $\hat{\mu}(x)$ to a function analytic on the strip $\left\{x: \operatorname{Re} x \in\left(c_{1}, c_{2}\right)\right\}$ in the complex plane. If

$$
\int e^{c|y|} \mu(d y)<\infty
$$

for some $c>0$, then the value of $\hat{\mu}(x)$ on any interval containing the origin uniquely determines $\mu$ and $\hat{\mu}(x)$ is called the moment generating function of $\mu$. If, further, $\hat{\mu}(x)$ is extended to a meromorphic function on the complex plane, the extension is denoted by the same symbol $\hat{\mu}(x)$ and still called the moment generating function of $\mu$. For any nonzero real number $a$, the exponential distribution $\mu_{a}$ has moment generating function

$$
\hat{\mu}_{a}(x)=a /(a-x) .
$$

For any $\mu$ in $E(k, l, r, s)$, there is $c>0$ such that (3.4) holds. The following facts are known.

LEMMA 3.1. Let $k \geq 1$. If $\mu \in M E_{++}^{k}$ is such that (3.1) holds with

$$
0<a_{1}<\cdots<a_{k},
$$

then $\mu$ has moment generating function

$$
\hat{\mu}(x)=\frac{a_{1} a_{2} \cdots a_{k}}{b_{1} b_{2} \cdots b_{k-1}} \frac{\left(b_{1}-x\right)\left(b_{2}-x\right) \cdots\left(b_{k-1}-x\right)}{\left(a_{1}-x\right)\left(a_{2}-x\right) \cdots\left(a_{k}-x\right)}
$$

for some $b_{1}, \ldots, b_{k-1}$ satisfying

$$
0<a_{1}<b_{1}<a_{2}<b_{2}<\cdots<a_{k-1}<b_{k-1}<a_{k} .
$$

Conversely, any rational function of the form of the right-hand side of (3.7) satisfying (3.8) is the moment generating function of some $\mu \in M E_{++}^{k}$ such that (3.1) holds with some $p_{1}, \ldots, p_{k}$.

LEMMA 3.2. Let $k \geq 1$. If $\mu \in M E_{+0}^{k}$ satisfies (3.2) and (3.6), then $\mu$ has moment generating function

$$
\hat{\mu}(x)=\frac{a_{1} \cdots a_{k}}{b_{1} \cdots b_{k}} \frac{\left(b_{1}-x\right) \cdots\left(b_{k}-x\right)}{\left(a_{1}-x\right) \cdots\left(a_{k}-x\right)}
$$

for some $b_{1}, \ldots, b_{k}$ such that

$$
0<a_{1}<b_{1}<a_{2}<b_{2}<\cdots<a_{k}<b_{k} .
$$

Any rational function of the form of the right-hand side of (3.9) satisfying (3.10) is the moment generating function of some $\mu \in M E_{+0}^{k}$ satisfying (3.2) for some $p_{0}, \ldots, p_{k}$.

Similar assertions hold for $M E_{--}^{k}$ and $M E_{-0}^{k}$. The distribution (3.1) with the condition

$$
a_{1}<\cdots<a_{k}<0
$$


corresponds to the moment generating function (3.7) combined with the condition

$$
a_{1}<b_{1}<a_{2}<b_{2}<\cdots<a_{k-1}<b_{k-1}<a_{k}<0 .
$$

The distribution (3.2) together with the condition (3.11) corresponds to the moment generating function (3.9) satisfying

$$
b_{1}<a_{1}<b_{2}<a_{2}<\cdots<b_{k}<a_{k}<0 .
$$

Proofs of Lemmas 3.1 and 3.2 are given in Steutel [14]. These lemmas show that the classes $M E_{++}^{k}(k=1,2, \ldots)$ are disjoint, and so are the classes $M E_{+0}^{k}(k=$ $0,1, \ldots)$. Moreover, if $\mu \in M E_{++}^{k}$ [resp. $M E_{--}^{k}$ ], then the numbers $a_{1}, b_{1}, a_{2}, \ldots$, $b_{k-1}, a_{k}$ satisfying (3.8) [resp. (3.12)] are uniquely determined in expressing the moment generating function of $\mu$. We call these numbers the parameters of $\mu$. Similarly, if $\mu \in M E_{+0}^{k}$ [resp. $M E_{-0}^{k}$ ], then the numbers $a_{1}, b_{1}, \ldots, a_{k}, b_{k}$ satisfying (3.10) [resp. (3.13)] are uniquely determined, which we call the parameters of $\mu$. If $\mu \in C E_{+}^{k}$, then

$$
\hat{\mu}(x)=\frac{a_{1} \cdots a_{k}}{\left(a_{1}-x\right) \cdots\left(a_{k}-x\right)}
$$

for $a_{1}, \ldots, a_{k}$ satisfying

$$
0<a_{1} \leq a_{2} \leq \cdots \leq a_{k},
$$

and conversely. If $\mu \in C E_{-}^{k}$, then we have (3.14) for $a_{1}, \ldots, a_{k}$ satisfying

$$
a_{1} \leq a_{2} \leq \cdots \leq a_{k}<0,
$$

and conversely. These numbers are called the parameters of $\mu$ in $C E_{+}^{k}$ or $C E_{-}^{k}$.

4. Distributions of sojourn times for birth-and-death processes. Let $X(t)$ be a birth-and-death process with birth rate $\alpha_{i}$ and death rate $\beta_{i}$. That is, $X(t)$ is a time homogeneous Markov process on the set of nonnegative integers with $Q$-matrix $\left\{q_{i j}\right\}$ such that

$$
\begin{gathered}
q_{i, i+1}=\alpha_{i} \quad(i \geq 0), \quad q_{i, i-1}=\beta_{i} \quad(i \geq 1), \\
q_{00}=-\alpha_{0}, \quad q_{i i}=-\alpha_{i}-\beta_{i} \quad(i \geq 1) \\
q_{i j}=0 \quad \text { otherwise. }
\end{gathered}
$$

We assume that $\alpha_{i}>0$ for $i \geq 0$ and $\beta_{i}>0$ for $i \geq 1$. The sample path of $X(t)$ is a right-continuous step function with jumps of size +1 or -1 . Let $\sigma^{m n}$ be the first time at which $X(t)=n$, given that $X(0)=m$. That is, $\sigma^{m n}$ is the first passage time to $n$ from $m$. Given a real-valued function $f(i)$ on the set of nonnegative integers, consider a random variable $T$ defined by

$$
T=\int_{0}^{\sigma^{m n}} f(X(t)) d t
$$

Denote by $\tau_{i}^{m n}$ the amount of time spent at the state $i$ up to the first passage time $\sigma^{m n}$. This quantity is called the sojourn time at $i$ up to $\sigma^{m n}$. From now on, we assume that $m<n$. The quantity $T$ is written as

$$
T=\sum_{i=0}^{n-1} f(i) \tau_{i}^{m n} .
$$


We call $T$ the sojourn time up to $\sigma^{m n}$ with weight function $f(i)$. We will determine the distribution $\mu$ of $T$. Let $N^{+}(n)$ [resp. $N^{-}(n)$ ] be the number of $i$ such that $0 \leq i \leq n-1$ and $f(i)>0$ [resp. $f(i)<0$ ]. Let $N(n)=N^{+}(n)+N^{-}(n)$. Then $n-N(n)$ is the number of $i$ such that $0 \leq i \leq n-1$ and $f(i)=0$. In order to state our results, we use the following notations for some subclasses of $E(k, l, r, s)$ :

$$
\begin{aligned}
E\left((k)_{++}, l,(r)_{-0}, s\right) & =M E_{++}^{k} * C E_{+}^{l} * M E_{-0}^{r} * C E_{-}^{s}, \\
E\left((k)_{+0}, l,(r)_{--}, s\right) & =M E_{+0}^{k} * C E_{+}^{l} * M E_{--}^{r} * C E_{-}^{s}, \\
E\left((k)_{+0}, l,(r)_{-0}, s\right) & =M E_{+0}^{k} * C E_{+}^{l} * M E_{-0}^{r} * C E_{-}^{s} .
\end{aligned}
$$

THEOREM 4.1. Let

$$
\begin{array}{ll}
k=N^{+}(m+1), & l=N^{+}(n)-N^{+}(m+1), \\
r=N^{-}(m+1), & s=N^{-}(n)-N^{-}(m+1) .
\end{array}
$$

Then the distribution $\mu$ of $T$ belongs to the class $E(k, l, r, s)$. More precisely,

$$
\begin{aligned}
& \mu \in E\left((k)_{++}, l,(r)_{-0}, s\right) \quad \text { in case } f(m)>0 ; \\
& \mu \in E\left((k)_{+0}, l,(r)_{--}, s\right) \quad \text { in case } f(m)<0 ; \\
& \mu \in E\left((k)_{+0}, l,(r)_{-0}, s\right) \quad \text { in case } f(m)=0 .
\end{aligned}
$$

Combining Theorem 4.1 with the results stated in $\S 3$ and using Lemma 6.1 of [12] or Lemma 3 of $[\mathbf{1 1}]$, we obtain the following results.

COROLLARY. The distribution $\mu$ is infinitely divisible and strictly unimodal. It has real-analytic densities on $(-\infty, 0)$ and $(0,+\infty)$. Let a be the mode of $\mu$. If $n=m+1$, then $a=0$. If $n \geq m+2$ and $f(i)=0$ for all $i$ such that $n<i<m$, then $a=0$. If $n \geq m+2$ and $f(i) \geq 0$ [resp. $f(i) \leq 0]$ for all $i$ such that $n<i<m$, then $a \geq 0$ [resp. $a \leq 0]$. The distribution $\mu$ has a point mass at 0 if and only if $f(i)=0$ for all $i$ satisfying $m \leq i<n$.

We note that infinite divisibility of $\mu$ is already proved by Kent [6]. In fact he proves that the joint distribution of $\left(\tau_{0}^{m n}, \tau_{1}^{m n}, \ldots, \tau_{n-1}^{m n}\right)$ is infinitely divisible. If $f$ is a constant function 1 , then $T=\sigma^{m n}$. Unimodality of first passage times is proved by Rösler [8] and Keilson [5]. If $f$ is positive, then $T$ can be considered via time change as a first passage time of another birth-and-death process. The exact class of first passage time distributions for birth-and-death processes is determined by Yamazato [16]. Our method of proof of Theorem 4.1, as well as Theorem 4.2 below, is a generalization of Yamazato's in [16].

In some cases it is possible to reduce the numbers $k, r$ of the class $E(k, l, r, s)$ to which $\mu$ belongs.

THEOREM 4.2. Let $l=N^{+}(n)-N^{+}(m+1)$ and $s=N^{-}(n)-N^{-}(m+1)$. There exist nonnegative integers $k$ and $r$ that have the following properties (i)-(iv). The pair $k, r$ that satisfies (i) and (ii) is unique; the pair that satisfies (i) and (iii) is unique.

(i) (4.3) -(4.5) hold.

(ii) If $k^{\prime}+l^{\prime}<k+l$ or $r^{\prime}+s^{\prime}<r+s$, then $\mu$ does not belong to $E\left(k^{\prime}, l^{\prime}, r^{\prime}, s^{\prime}\right)$.

(iii) If $\mu^{(1)} * \mu^{(2)} * \mu^{(3)} * \mu^{(4)}$ is an expression of $\mu$ in the class described in (4.3)(4.5), then the set of all parameters of $\mu^{(1)}, \mu^{(2)}, \mu^{(3)}$, and $\mu^{(4)}$ has no overlapping. 
(iv)

$$
\begin{aligned}
& \max \left\{2 N^{+}(m+1)-N^{+}(n), N^{+}(m+1)-N^{+}(m)\right\} \leq k \leq N^{+}(m+1), \\
& \max \left\{2 N^{-}(m+1)-N^{-}(n), N^{-}(m+1)-N^{-}(m)\right\} \leq r \leq N^{-}(m+1) .
\end{aligned}
$$

Notice that a distribution in $E(k, l, r, s)$ does not necessarily have a unique expression. Thus the identity $\mu^{(1)} * \mu^{(2)}=\nu^{(1)} * \nu^{(2)}$ with $\mu^{(1)}, \nu^{(1)} \in M E_{++}^{k}$ and $\mu^{(2)}, \nu^{(2)} \in C E_{+}^{l}$ does not imply $\mu^{(1)}=\nu^{(1)}$ and $\mu^{(2)}=\nu^{(2)}$.

5. Proofs of Theorems 2.1-2.3. For $n \geq 0$ and $0 \leq k \leq n$, define $D_{k}^{n}$ as follows:

$$
\left\{\begin{array}{l}
D_{0}^{n}=1, \quad D_{1}^{n}=B_{n-1}, \\
D_{k}^{n}=B_{n-k} D_{k-1}^{n}-C_{n-k+1} D_{k-2}^{n} \quad \text { for } 2 \leq k \leq n .
\end{array}\right.
$$

LEMMA 5.1. The $D_{k}^{n}$ have the properties below.

$$
\begin{aligned}
& P_{n}(0)=D_{k}^{n} P_{n-k}(0)-C_{n-k} D_{k-1}^{n} P_{n-k-1}(0) \quad \text { for } 1 \leq k \leq n-1, \\
& P_{n}(0)=D_{n}^{n} \quad \text { for } n \geq 0, \\
& D_{k}^{n}=B_{n-1} D_{k-1}^{n-1}-C_{n-1} D_{k-2}^{n-2} \quad \text { for } 2 \leq k \leq n .
\end{aligned}
$$

PROOF. First we prove (5.2) by induction in $k$. If $k=1$, then (5.2) is true for all $n \geq 2$ by (1.1), because

$$
P_{n}(0)=B_{n-1} P_{n-1}(0)-C_{n-1} P_{n-2}(0)=D_{1}^{n} P_{n-1}(0)-C_{n-1} D_{0}^{n} P_{n-2}(0) .
$$

If (5.2) is true for a given $k$ and all $n \geq k+1$, then

$$
\begin{aligned}
P_{n}(0) & =D_{k}^{n}\left(B_{n-k-1} P_{n-k-1}(0)-C_{n-k-1} P_{n-k-2}(0)\right)-C_{n-k} D_{k-1}^{n} P_{n-k-1}(0) \\
& =D_{k+1}^{n} P_{n-k-1}(0)-C_{n-k-1} D_{k}^{n} P_{n-k-2}(0)
\end{aligned}
$$

for $n \geq k+2$ by (5.1). Thus (5.2) is proved. Since $P_{0}(0)=1$ and $P_{1}(0)=B_{0}$, (5.3) is obvious for $n=0$ and 1 . Hence, using (5.2), we have $P_{n}(0)=D_{n-1}^{n} P_{1}(0)-$ $C_{1} D_{n-2}^{n} P_{0}(0)=D_{n}^{n}$ for $n \geq 2$, that is (5.3) holds. The assertion (5.4) is proved by induction in $k$. If $n \geq 2$, then

$$
D_{2}^{n}=B_{n-2} D_{1}^{n}-C_{n-1} D_{0}^{n}=B_{n-1} B_{n-2}-C_{n-1}=B_{n-1} D_{1}^{n-1}-C_{n-1} D_{0}^{n-2} .
$$

If $n \geq 3$, then

$$
\begin{aligned}
D_{3}^{n} & =B_{n-3} D_{2}^{n}-C_{n-2} D_{1}^{n}=\left(B_{n-1} D_{1}^{n-1}-C_{n-1} D_{0}^{n-2}\right) B_{n-3}-B_{n-1} C_{n-2} \\
& =B_{n-1}\left(B_{n-3} D_{1}^{n-1}-C_{n-2}\right)-C_{n-1} B_{n-3}=B_{n-1} D_{2}^{n-1}-C_{n-1} D_{1}^{n-2} .
\end{aligned}
$$

Thus (5.4) is true for $k=2,3$. Let $l \geq 3$. If (5.4) is true for $k=l-1$ and $l$, then it is true for $k=l+1$, because

$$
\begin{aligned}
D_{l+1}^{n} & =B_{n-l-1} D_{l}^{n}-C_{n-l} D_{l-1}^{n} \\
& =\left(B_{n-1} D_{l-1}^{n-1}-C_{n-1} D_{l-2}^{n-2}\right) B_{n-l-1}-\left(B_{n-1} D_{l-2}^{n-1}-C_{n-1} D_{l-3}^{n-2}\right) C_{n-l} \\
& =B_{n-1}\left(B_{n-l-1} D_{l-1}^{n-1}-C_{n-l} D_{l-2}^{n-1}\right)-C_{n-1}\left(B_{n-l-1} D_{l-2}^{n-2}-C_{n-l} D_{l-3}^{n-2}\right) \\
& =B_{n-1} D_{l}^{n-1}-C_{n-1} D_{l-1}^{n-2} .
\end{aligned}
$$

Hence (5.4) is established. 
LEMMA 5.2. $D_{k}^{n}>0$ for $0 \leq k \leq n$.

PROOF. This is obvious for $k=0,1$. If, for a given $k, D_{k-1}^{n}>0$ for all $n \geq k-1$, then (5.2), (A.1), and (A.2) show that $D_{k}^{n}>0$ for $n \geq k+1$. Moreover $D_{k}^{k}>0$ by (5.3) and (A.2).

Lemma 5.3. (i) The degree of $P_{n}(x)$ is $N(n)$.

(ii) Given $n \geq 2$, suppose that $A_{n-1}=A_{n-2}=\cdots=A_{n-k}=0$ and $A_{n-k-1} \neq 0$ for some $1 \leq k \leq n-1$. Let $\gamma$ be the highest coefficient of $P_{n-k}(x)$. Then $P_{n}(x)$ has $D_{k}^{n} \gamma$ as its highest coefficient. $D_{n}^{n}$.

(iii) Given $n \geq 1$, suppose that $A_{n-1}=A_{n-2}=\cdots=A_{0}=0$. Then $P_{n}(x)=$

ProOF. For $n=1$, (i) and (iii) are obvious. For $n=2$, (i), (ii), and (iii) are easy to check. Let $m \geq 3$ and suppose that (i), (ii), (iii) are true for $n \leq m-1$. We have

$$
P_{m}(x)=\left(B_{m-1}-A_{m-1} x\right) P_{m-1}(x)-C_{m-1} P_{m-2}(x)
$$

and $P_{m-1}$ and $P_{m-2}$ are of degree $N(m-1)$ and $N(m-2)$, respectively. If $A_{m-1} \neq 0$, then $\operatorname{deg} P_{m}=N(m-1)+1=N(m)$. If $A_{m-1}=\cdots=A_{m-k}=0$ and $A_{m-k-1} \neq 0$ for some $1 \leq k \leq m-1$, then consider two cases: $k=1$ and $k \geq 2$. In case $k=1$, we have $N(m-1)=N(m-2)+1$ and $\operatorname{deg} P_{m}=N(m-1)=N(m)$ and the highest coefficient of $P_{m}$ is $B_{m-1} \gamma=D_{1}^{m} \gamma$. In case $k \geq 2, P_{m-1}$ and $P_{m-2}$ are of a common degree with their highest coefficients being $D_{k-1}^{m-1} \gamma$ and $D_{k-2}^{m-2} \gamma$, respectively, and hence, by (5.4) and by Lemma $5.2, P_{m}$ is of degree $N(m-1)=$ $N(m)$ and has $D_{k}^{m} \gamma$ as its highest coefficient. If $A_{m-1}=\cdots=A_{1}=A_{0}=0$, then $P_{m-1}(x)=D_{m-1}^{m-1}, P_{m-2}(x)=D_{m-2}^{m-2}$, and

$$
P_{m}(x)=B_{m-1} D_{m-1}^{m-1}-C_{m-1} D_{m-2}^{m-2}=D_{m}^{m}>0
$$

by Lemma 5.1 and (A.2). The proof is complete.

After proving Theorem 2.1(i), we will show Theorems 2.1(ii) and 2.3 similtaneously, and then, using these theorems, prove Theorem 2.2.

ProOF OF THEOREM 2.1(i). The fact $\operatorname{deg} P_{n}=N(n)$ is given in Lemma 5.3(i). If $A_{n} \neq 0$, then $\operatorname{deg} P_{n+1}=\operatorname{deg} P_{n}+1$ and, by (1.1), the highest coefficient of $P_{n+1}$ is $-A_{n}$ times the highest coefficient of $P_{n}$. If $A_{n}=0$, then by Lemmas 5.2 and 5.3, the highest coefficient of $P_{n+1}$ and that of $P_{n}$ have a common sign. Hence, for any $n$, the sign of the highest coefficient of $P_{n}$ is $(-1)^{N^{+}(n)}$.

PROOF OF THEOREM 2.1(ii) AND THEOREM 2.3. We do induction on $n$. We will repeatedly use (1.1), (A.1), and (A.2). In particular, the following fact is essential.

$$
\text { If } P_{n}(a)=0, \text { then } \operatorname{sgn} P_{n+1}(a)=-\operatorname{sgn} P_{n-1}(a) .
$$

Here we are using the signum function $\operatorname{sgn} x$ defined by $\operatorname{sgn} x=-1,0,+1$ according to whether $x$ is negative, zero, or positive. Also we will use the following consequences of Theorem 2.1(i).

$$
\operatorname{sgn} P_{n}(-\infty)=(-1)^{N^{-}(n)}, \quad \operatorname{sgn} P_{n}(+\infty)=(-1)^{N^{+}(n)} .
$$


Let $\alpha$ and $\beta$ be defined by $\operatorname{sgn} P_{n}(-\infty)=\alpha \operatorname{sgn} P_{n-1}(-\infty)$ and $\operatorname{sgn} P_{n}(+\infty)=$ $\beta$ sgn $P_{n-1}(+\infty)$. Then,

$$
\left\{\begin{array}{l}
A_{n-1}>0 \text { implies } \alpha=+1, \quad \beta=-1, \\
A_{n-1}<0 \text { implies } \alpha=-1, \quad \beta=+1, \\
A_{n-1}=0 \text { implies } \alpha=+1, \quad \beta=+1 .
\end{array}\right.
$$

If it is known that $P_{n}(x)$ has $N(n)$ simple real zeros, then the zeros are denoted by $b_{1}^{n}<b_{2}^{n}<\cdots<b_{N(n)}^{n}$.

Let us consider $P_{1}(x)$ and $P_{2}(x)$ in three cases.

(i) Case $A_{0}>0$. We have $\operatorname{deg} P_{1}=1$ and $0=a_{1}^{1}<a_{2}^{1}$. Note that $P_{2}(0)>0$ and $P_{2}\left(a_{2}^{1}\right)<0$. If $A_{1}>0$, then $\operatorname{deg} P_{2}=2, P_{2}(+\infty)>0$, and thus $0=a_{1}^{1}<b_{1}^{2}<a_{2}^{1}<$ $b_{2}^{2}$. If $A_{1}<0$, then $\operatorname{deg} P_{2}=2, P_{2}(-\infty)<0$, and hence $b_{1}^{2}<0=a_{1}^{1}<b_{2}^{2}<a_{2}^{1}$. If $A_{1}=0$, then $\operatorname{deg} P_{2}=1$ and $0=a_{1}^{1}<b_{1}^{2}<a_{2}^{1}$.

(ii) Case $A_{0}<0$. We have $\operatorname{deg} P_{1}=1, a_{1}^{1}<a_{2}^{1}=0, P_{2}\left(a_{1}^{1}\right)<0$, and $P_{2}(0)>0$. If $A_{1}>0$, then $\operatorname{deg} P_{2}=2, P_{2}(+\infty)<0$, and $a_{1}^{1}<b_{1}^{2}<a_{2}^{1}=0<b_{2}^{2}$. If $A_{1}<0$, then $\operatorname{deg} P_{2}=2, P_{2}(-\infty)>0$, and $b_{1}^{2}<a_{1}^{1}<b_{2}^{2}<a_{2}^{1}=0$. If $A_{1}=0$, then $\operatorname{deg} P_{2}=1$ and $a_{1}^{1}<b_{1}^{2}<a_{2}^{1}=0$.

(iii) Case $A_{0}=0$. We have $P_{1}(x)=B_{0}>0$ and $P_{2}(0)>0$. If $A_{1}>0$, then $\operatorname{deg} P_{2}=1$ and $b_{1}^{2}>0$. If $A_{1}<0$, then $\operatorname{deg} P_{2}=1$ and $b_{1}^{2}<0$. If $A_{1}=0$, then $\operatorname{deg} P_{2}=0$.

The above discussion shows that Theorem 2.1(ii) holds for $n=1,2$ and Theorem 2.3 holds for $m=1$. Given $k \geq 2$, suppose that Theorems 2.1(ii) and 2.3 are true for $n \leq k$ and $m=k-1$, respectively. We will prove them for $n=k+1$ and $m=k$. Let $i_{0}$ be the number such that $a_{i_{0}}^{k}=0$.

$1^{\circ}$ Let $i \leq i_{0}-2$ or $i \geq i_{0}+1$. Then $P_{k-1}$ has exactly one zero between $a_{i}^{k}$ and $a_{i+1}^{k}$, and thus $\operatorname{sgn} P_{k-1}\left(a_{i}^{k}\right)=-\operatorname{sgn} P_{k-1}\left(a_{i+1}^{k}\right) \neq 0$. Hence $\operatorname{sgn} P_{k+1}\left(a_{i}^{k}\right)=$ $-\operatorname{sgn} P_{k+1}\left(a_{i+1}^{k}\right) \neq 0$ by (5.5). Therefore $P_{k+1}$ has a zero between $a_{i}^{k}$ and $a_{i+1}^{k}$.

$2^{\circ}$ Suppose that $2 \leq i_{0} \leq N(k)$. If $A_{k-1} \geq 0$, then we have $a_{i_{0}-1}^{k-1}<a_{i_{0}-1}^{k}<$ $a_{i_{0}}^{k-1}=a_{i_{0}}^{k}=0<a_{i_{0}+1}^{k}$ (and further $a_{i_{0}+1}^{k}<a_{i_{0}+1}^{k-1}$ except the case where $i_{0}=$ $N(k)$ and $A_{k-1}>0$ ), and hence $P_{k-1}$ is positive at $a_{i_{0}-1}^{k}, a_{i_{0}}^{k}$, and $a_{i_{0}+1}^{k}$, implying

$$
P_{k+1}\left(a_{i_{0}-1}^{k}\right)<0, \quad P_{k+1}\left(a_{i_{0}}^{k}\right)>0, \quad P_{k+1}\left(a_{i_{0}+1}^{k}\right)<0
$$

by (5.5) and (A.2). If $A_{k-1}<0$, then $a_{i_{0}-1}^{k}<a_{i_{0}-1}^{k-1}=a_{i_{0}}^{k}=0<a_{i_{0}+1}^{k}<a_{i_{0}}^{k-1}$ (and $a_{i_{0}-2}^{k-1}<a_{i_{0}-1}^{k}$ except the case $i_{0}=2$ ), and (5.8) holds by the same reason. Thus $P_{k+1}$ has zeros in $\left(a_{i_{0}-1}^{k}, a_{i_{0}}^{k}\right)$ and in $\left(a_{i_{0}}^{k}, a_{i_{0}+1}^{k}\right)$.

$3^{\circ}$ Suppose that $i_{0}=1$. Then $A_{k-1} \geq 0$ and $a_{1}^{k-1}=a_{1}^{k}=0<a_{2}^{k}<a_{2}^{k-1}<a_{3}^{k}<$ $a_{3}^{k-1}$. Thus $P_{k-1}$ is positive at $a_{1}^{k}$ and $a_{2}^{k}$. Hence $P_{k+1}\left(a_{1}^{k}\right)>0$ and $P_{k+1}\left(a_{2}^{k}\right)<0$. Thus $P_{k+1}$ has a zero between $a_{i_{0}}^{k}$ and $a_{i_{0}+1}^{k}$.

$4^{\circ}$ Suppose that $i_{0}=N(k)+1$. Then, similarly, $P_{k+1}$ has a zero between $a_{i_{0}-1}^{k}$ and $a_{i_{0}}^{k}$.

The above argument shows that the points $a_{1}^{k}, a_{2}^{k}, \ldots, a_{N(k)+1}^{k}$ are separated by $N(k)$ zeros of $P_{k+1}$. If $A_{k}=0$, then $N(k+1)=N(k)$ and $P_{k+1}$ has no other zero. Let us consider the case $A_{k}>0$. We will prove that $P_{k+1}$ has a zero in the interval $\left(a_{N(k)+1}^{k},+\infty\right)$, by showing

$$
\operatorname{sgn} P_{k+1}\left(a_{N(k)+1}^{k}\right)=-\operatorname{sgn} P_{k+1}(+\infty) .
$$


Denote, for a while, $\alpha_{n}=\operatorname{sgn} P_{n}(+\infty)$. We have to consider four cases.

Case 1. $\quad \alpha_{k}=-1, A_{k-1}>0$ (hence $\alpha_{k-1}=+1, \alpha_{k+1}=+1$ by (5.7)).

Case 2. $\alpha_{k}=-1, A_{k-1} \leq 0$ (hence $\alpha_{k-1}=-1, \alpha_{k+1}=+1$ ).

Case 3. $\alpha_{k}=+1, A_{k-1}>0$ (hence $\alpha_{k-1}=-1, \alpha_{k+1}=-1$ ).

Case 4. $\alpha_{k}=+1, A_{k-1} \leq 0$ (hence $\alpha_{k-1}=+1, \alpha_{k+1}=-1$ ).

Case 1. We have $a_{N(k-1)+1}^{k-1}<b_{N(k)}^{k}=a_{N(k)+1}^{k}$ and hence

$$
P_{k-1}\left(a_{N(k)+1}^{k}\right)>0 \text { and } P_{k+1}\left(a_{N(k)+1}^{k}\right)<0
$$

by (5.5), from which follows (5.9).

Case 2. We have $b_{N(k)}^{k}<a_{N(k-1)+1}^{k-1}$. Hence $a_{N(k-1)+1}^{k-1} \neq 0, b_{N(k)}^{k}=a_{N(k)+1}^{k}$, and (5.10) follows. Hence (5.9).

Case 3. This time $a_{N(k-1)+1}^{k-1}<b_{N(k)}^{k}=a_{N(k)+1}^{k}$ and

$$
P_{k-1}\left(a_{N(k)+1}^{k}\right)<0 \text { and } P_{k+1}\left(a_{N(k)+1}^{k}\right)>0,
$$

guaranteeing (5.9).

Case 4. We have $b_{N(k)}^{k}<a_{N(k-1)+1}^{k-1}$. If $a_{N(k-1)+1}^{k-1}=0$, then $a_{N(k)+1}^{k}=0$ and (5.9) follows from (A.2). If $a_{N(k-1)+1}^{k-1} \neq 0$, then $a_{N(k)+1}^{k}=b_{N(k)}^{k} \neq 0$ and we have (5.11), which shows (5.9).

In conclusion, (5.9) is shown in all cases. It is proved similarly that, if $A_{k}<0$, then $P_{k+1}$ has a zero in $\left(-\infty, a_{1}^{k}\right)$. Thus we have seen that $P_{k+1}$ has $N(k+1)$ real zeros and Theorem 2.3 is true for $n=k$. Therefore, if $A_{k}>0$, then the number of positive zeros of $P_{k+1}$ is larger than that of $P_{k}$ by one and the number of negative zeros of $P_{k+1}$ is the same as that of $P_{k}$. If $A_{k}<0$, then the number of negative zeros increases by one. If $A_{k}=0$, the number of positive zeros and that of negative zeros do not change. Namely, Theorem 2.1(ii) is true for $n=k+1$. The proof of Theorem 2.1(ii) and Theorem 2.3 is complete.

To proceed to the proof of Theorem 2.2, we introduce a new system of polynomials $Q_{0}^{n}, \ldots, Q_{n}^{n}$, following Yamazato. This is in a sense adjoint to the system $P_{0}, \ldots, P_{n}$. Define

$$
\left\{\begin{array}{l}
Q_{0}^{n}(x)=1 \text { for } n \geq 0, \\
Q_{1}^{n}(x)=B_{n-1}-A_{n-1} x \text { for } n \geq 1, \\
Q_{k+1}^{n}(x)=\left(B_{n-k-1}-A_{n-k-1} x\right) Q_{k}^{n}(x)-C_{n-k} Q_{k-1}^{n}(x) \text { for } 1 \leq k \leq n-1 .
\end{array}\right.
$$

LEMMA 5.4. For $n \geq 2$ and $1 \leq k \leq n-1$,

$$
\begin{aligned}
& P_{n}(x)=Q_{k}^{n}(x) P_{n-k}(x)-C_{n-k} Q_{k-1}^{n}(x) P_{n-k-1}(x), \\
& P_{n}(x)=Q_{n}^{n}(x) .
\end{aligned}
$$

ProOF. Let us show (5.13) by induction. It is true for $k=1$ by (1.1). Suppose that $n \geq 3$ and that (5.13) holds for some $k \leq n-2$. Then,

$$
\begin{aligned}
P_{n} & =Q_{k}^{n}\left(\left(B_{n-k-1}-A_{n-k-1} x\right) P_{n-k-1}-C_{n-k-1} P_{n-k-2}\right)-C_{n-k} Q_{k-1}^{n} P_{n-k-1} \\
& =Q_{k+1}^{n} P_{n-k-1}-C_{n-k-1} Q_{k}^{n} P_{n-k-2},
\end{aligned}
$$

which is (5.13) with $k$ replaced by $k+1$. Hence (5.13) is true for all $k \leq n-1$. We obtain (5.14) by

$$
P_{n}=Q_{n-1}^{n} P_{1}-C_{1} Q_{n-2}^{n} P_{0}=\left(B_{0}-A_{0} x\right) Q_{n-1}^{n}-C_{1} Q_{n-2}^{n}=Q_{n}^{n} .
$$


LEMMA 5.5. $Q_{k}^{n}(0)>0$ for $0 \leq k \leq n$.

ProOF. The assertion is evident for $n=0,1$. Let $n \geq 2$. We have $Q_{0}^{n}(0)=1>$ 0 . Noting (5.13), (A.1), and (A.2), we see that $Q_{k-1}^{n}(0)>0$ implies $Q_{k}^{n}(0)>0$.

Rewriting (5.13) we get

$$
P_{n}(x)=Q_{n-m-1}^{n}(x) P_{m+1}(x)-C_{m+1} Q_{n-m-2}^{n}(x) P_{m}(x) \text { for } 0 \leq m \leq n-2 .
$$

Hence we obtain the following.

LEMMA 5.6. For $0 \leq m \leq n-2$,

$$
\begin{gathered}
\frac{P_{n}(x)}{P_{m}(x)}=Q_{n-m-1}^{n}(x)\left(\frac{P_{m+1}(x)}{P_{m}(x)}-C_{m+1} \frac{Q_{n-m-2}^{n}(x)}{Q_{n-m-1}^{n}(x)}\right), \\
C_{m+1} \frac{Q_{n-m-2}^{n}(0)}{Q_{n-m-1}^{n}(0)}<\frac{P_{m+1}(0)}{P_{m}(0)} .
\end{gathered}
$$

PROOF OF THEOREM 2.2. If $n=m+1$, then the theorem is already proved by Theorem 2.3. So, let $0 \leq m \leq n-2$. Let

$$
f(x)=\frac{P_{m+1}(x)}{P_{m}(x)}, \quad g(x)=C_{m+1} \frac{Q_{n-m-2}^{n}(x)}{Q_{n-m-1}^{n}(x)} .
$$

In order to seek zeros of the right-hand side of (5.16), we investigate the graphs of $f(x)$ and $g(x)$ in detail by using Theorems 2.1 and 2.3. We study first the graph of $f(x)$. On an interval with endpoints being consecutive zeros of $P_{m}$ or $+\infty$ or $-\infty$, the behavior of $f(x)$ has eight possibilities:

(5.18) increase from $-\infty$ to $+\infty$,

(5.19) decrease from $+\infty$ to $-\infty$,

(5.20) increase from a finite $c$ to $+\infty$,

(5.21) decrease from a finite $c$ to $-\infty$,

(5.22) increase from $-\infty$ to a finite $c$,

(5.23) decrease from $+\infty$ to a finite $c$,

(5.24) increase from $-\infty$ to a positive local maximum and then decrease to $-\infty$,

(5.25) decrease from $+\infty$ to a positive local minimum and then increase to $+\infty$.

Let $i_{0}$ be the number such that $a_{i_{0}}^{m}=0$. There are three cases.

Case 1. $2 \leq i_{0} \leq N(m)$. The numerator $P_{m+1}$ has a zero in each of the intervals $\left(a_{i_{0}-1}^{m}, a_{i_{0}}^{m}\right)$ and $\left(a_{i_{0}}^{m}, a_{i_{0}+1}^{m}\right)$. We have $f(0)>0$. Hence (5.24) holds on $\left(a_{i_{0}-1}^{m}, a_{i_{0}+1}^{m}\right)$. For $1 \leq i \leq i_{0}-2$ we have $(5.18)$ on $\left(a_{i}^{m}, a_{i+1}^{m}\right)$. For $i_{0}+1 \leq i \leq$ $N(m),(5.19)$ holds on $\left(a_{i}^{m}, a_{i+1}^{m}\right)$. Further, there are three subcases.

Case 1a. $A_{m}>0$. We have (5.25) on $\left(-\infty, a_{1}^{m}\right)$ and (5.19) on $\left(a_{N(m)+1}^{m},+\infty\right)$.

Case 1b. $A_{m}<0$. We have (5.18) on $\left(-\infty, a_{1}^{m}\right)$ and (5.25) on $\left(a_{N(m)+1}^{m},+\infty\right)$.

Case 1c. $A_{m}=0$. There is a common positive number $c$ such that (5.20) holds on $\left(-\infty, a_{1}^{m}\right)$ and $(5.23)$ on $\left(a_{N(m)+1}^{m},+\infty\right)$. 
Note that monotonicity of $f(x)$ on each indicated interval comes from the fact that, for any $c$, the number of the roots of $f(x)=c$ is at most $N(m+1)$.

Case 2. $i_{0}=1$. First we consider $\left(-\infty, a_{2}^{m}\right)$ and proceed to the right. If $2 \leq i \leq N(m)$, then (5.19) holds on $\left(a_{i}^{m}, a_{i+1}^{m}\right)$.

Case 2a. $A_{m}>0$. On each of $\left(-\infty, a_{2}^{m}\right)$ and $\left(a_{N(m)+1}^{m},+\infty\right),(5.19)$ holds.

Case 2b. $A_{m}<0$. On $\left(-\infty, a_{2}^{m}\right)$ the behavior of $f(x)$ is (5.24) and, on $\left(a_{N(m)+1}^{m},+\infty\right),(5.25)$.

Case 2c. $A_{m}=0$. A positive number $c$ exists so that (5.21) holds on $\left(-\infty, a_{2}^{m}\right)$ and (5.23) on $\left(a_{N(m)+1}^{m},+\infty\right)$.

Case 3. $i_{0}=N(m)+1$. This is dual with Case 2. If $1 \leq i \leq N(m)-1$, then (5.18) holds on $\left(a_{i}^{m}, a_{i+1}^{m}\right)$.

Case 3a. $A_{m}>0$. On $\left(-\infty, a_{1}^{m}\right)$ and $\left(a_{N(m)}^{m},+\infty\right),(5.25)$ and (5.24) hold, respectively.

Case 3b. $A_{m}<0$. On each of $\left(-\infty, a_{1}^{m}\right)$ and $\left(a_{N(m)}^{m},+\infty\right),(5.18)$ holds.

Case 3c. $A_{m}=0$. There is $c>0$ such that (5.20) and (5.22) hold on $\left(-\infty, a_{1}^{m}\right)$ and $\left(a_{N(m)}^{m},+\infty\right)$, respectively.

Next we consider the graph of $g(x)$. By Definition (5.12) and Lemma 5.5, the polynomials $Q_{k}^{n}(x), 0 \leq k \leq n$, have the same properties as $P_{k}(x), 0 \leq k \leq$ $n$, except for change of defining constants. Thus the behavior of $1 / g(x)$ can be described as similar to that of $f(x)$. Since $A_{n-1}, A_{n-2}, \ldots, A_{0}$ play the role of $A_{0}, A_{1}, \ldots, A_{n-1}$, the degree of $Q_{k}^{n}$ is $N(n)-N(n-k)$. All zeros of $Q_{k}^{n}$ are real and simple. Suppressing $n$, we designate $N(n)-N(n-k)+1$ points consisting of the zeros of $Q_{k}^{n}$ and the point 0 as

$$
c_{1}^{k}<c_{2}^{k}<\cdots<c_{N(n)-N(n-k)+1}^{k} .
$$

Let $r=n-m-1$ and $s=\operatorname{deg} Q_{r}^{n}=N(n)-N(m+1)$. Let $j_{0}$ be the number such that $c_{j_{0}}^{r}=0$. The behavior of $g(x)$ is as follows.

Case 1. $2 \leq j_{0} \leq s$. On $\left(c_{j_{0}-1}^{r}, c_{j_{0}+1}^{r}\right)(5.25)$ holds. If $1 \leq j \leq j_{0}-2$, then (5.19) holds on $\left(c_{j}^{r}, c_{j+1}^{r}\right)$. If $j_{0}+1 \leq j \leq s$, then (5.18) holds on $\left(c_{j}^{r}, c_{j+1}^{r}\right)$.

Case 1a. $A_{m+1}>0$. On $\left(-\infty, c_{1}^{r}\right), g(x)$ increases from 0 to a positive local maximum and then decreases to $-\infty$. Further, we have (5.22) with $c=0$ on $\left(c_{s+1}^{r},+\infty\right)$.

Case 1b. $A_{m+1}<0$. We have (5.21) with $c=0$ on $\left(-\infty, c_{1}^{r}\right)$. On $\left(c_{s+1}^{r},+\infty\right)$, $g(x)$ increases from $-\infty$ to a positive local maximum and then decreases to 0 .

Case 1c. $A_{m+1}=0$. There is $c>0$ such that (5.21) holds on $\left(-\infty, c_{1}^{r}\right)$ and (5.22) holds on $\left(c_{s+1}^{r},+\infty\right)$.

Case 2. $j_{0}=1$. In this case we have $A_{m+1} \geq 0$. If $2 \leq j \leq s$, then (5.18) holds on $\left(c_{j}^{r}, c_{j+1}^{r}\right)$.

Case 2a. $A_{m+1}>0$. We have (5.20) with $c=0$ on $\left(-\infty, c_{2}^{r}\right)$ and (5.22) with $c=0$ on $\left(c_{s+1}^{r},+\infty\right)$.

Case $2 \mathrm{~b} . A_{m+1}=0$. There is $c>0$ satisfying (5.20) on $\left(-\infty, c_{2}^{r}\right)$ and (5.22) on $\left(c_{s+1}^{r},+\infty\right)$.

Case 3. $j_{0}=s+1$. In this case, $A_{m+1} \leq 0$. If $1 \leq j \leq s-1$, then (5.19) holds on $\left(c_{j}^{r}, c_{j+1}^{r}\right)$.

Case 3a. $A_{m+1}<0$. (5.21) holds on $\left(-\infty, c_{1}^{r}\right)$ and (5.23) holds on $\left(c_{s}^{r},+\infty\right)$, each with $c=0$. 
Case $3 \mathrm{~b} . A_{m+1}=0$. There is $c>0$ satisfying (5.21) on $\left(-\infty, c_{1}^{r}\right)$ and (5.23) on $\left(c_{s}^{r},+\infty\right)$.

Now let us prove (i) of Theorem 2.2 for $0 \leq m \leq n-2$. If $a$ satisfies

$$
P_{m}(a) \neq 0, \quad Q_{n-m-1}^{n}(a) \neq 0, \quad \frac{P_{m+1}(a)}{P_{m}(a)}=C_{m+1} \frac{Q_{n-m-2}^{n}(a)}{Q_{n-m-1}^{n}(a)},
$$

then $P_{n}(a)=0$ by $(5.16)$. For each $j(1 \leq j \leq N(m))$, we can prove that there is a point $a \in\left(a_{j}^{m}, a_{j+1}^{m}\right)$ for which (5.26) holds. Namely, in all combinations of the cases of the graph of $f(x)$ and the cases of the graph of $g(x)$, the graph of $f(x)$ for $a_{j}^{m}<x<a_{j+1}^{m}$ intersects with the graph of $g(x)$. When $a_{j}^{m}$ or $a_{j+1}^{m}$ is the point 0 , we use here Property (5.17). Also it can be proved that, if $A_{m}>0$, then there exists $a \in\left(a_{N(m)+1}^{m},+\infty\right)$ for which (5.26) holds. If $A_{m}<0$, then, similarly, we can find a point $a \in\left(-\infty, a_{1}^{m}\right)$ for which (5.26) is true. This proves (i) of Theorem 2.2 .

Let $k$ [resp. $k^{\prime}$ ] be the number of common positive [resp. negative] zeros of $P_{m}$ and $P_{n}$. Since $k$ [resp. $k^{\prime}$ ] does not exceed the number of positive [resp. negative] zeros of $P_{m}$, we have $k \leq N^{+}(m)$ and $k^{\prime} \leq N^{-}(m)$. By (5.15) and by Theorem 2.3, any common zero of $P_{n}$ and $P_{m}$ is a zero of $Q_{n-m-1}^{n}$. Since the number of positive [resp. negative] zeros of $Q_{n-m-1}^{n}$ is $N^{+}(n)-N^{+}(m+1)$ [resp. $N^{-}(n)-N^{-}(m+1)$ ], it follows that $k \leq N^{+}(n)-N^{+}(m+1)$ and $k^{\prime} \leq N^{-}(n)-N^{-}(m+1)$. The proof of Theorem 2.2 is complete.

6. Proofs of Theorems 4.1-4.2. We use the following two lemmas due to Kent [6]. A vector $\mathbf{x}=\left(x_{0}, x_{1}, \ldots\right)$ with infinitely many components is used, although each function of $\mathbf{x}$ introduced below depends only on a finite number of components. For $\mathbf{x}$ with $x_{i} \leq 0$ for all $i$, we define

$$
\phi_{m n}(\mathbf{x})=E \exp \sum_{i=0}^{n-1} \tau_{i}^{m n} x_{i}
$$

where $\tau_{i}^{m n}$ is the sojourn time at $i$ up to $\sigma^{m n}$ and $E$ denotes the expectation.

LEMMA 6.1. For $\mathbf{x}=\left(x_{0}, x_{1}, \ldots\right)$ with $x_{i} \leq 0$ for all $i$,

$$
\begin{gathered}
\alpha_{0} \phi_{1 n}(\mathbf{x})=\left(\alpha_{0}-x_{0}\right) \phi_{0 n}(\mathbf{x}), \\
\alpha_{i} \phi_{i+1, n}(x)=\left(\alpha_{i}+\beta_{i}-x_{i}\right) \phi_{i n}(\mathbf{x})-\beta_{i} \phi_{i-1, n}(\mathbf{x}) \quad \text { for } 1 \leq i \leq n-1, \\
\phi_{n n}(\mathbf{x})=1 .
\end{gathered}
$$

Proof is obtained from the strong Markov property of the process.

The following system of polynomials with many variables is introduced by Kent. This is a generalization of polynomials of Ledermann-Reuter [7] and KarlinMcGregor [3]. Define

$$
\left\{\begin{array}{l}
\tilde{P}_{0}(\mathbf{x})=1 \\
\tilde{P}_{1}(\mathbf{x})=1-x_{0} / \alpha_{0} \\
\tilde{P}_{i+1}(\mathbf{x})=\left(1+\beta_{i} / \alpha_{i}-x_{i} / \alpha_{i}\right) \tilde{P}_{i}(\mathbf{x})-\left(\beta_{i} / \alpha_{i}\right) \tilde{P}_{i-1}(\mathbf{x}) \text { for } i \geq 1
\end{array}\right.
$$

LEMMA 6.2. For $\mathbf{x}=\left(x_{0}, x_{1}, \ldots\right)$ with $x_{i} \leq 0$ for all $i$,

$$
\phi_{m n}(\mathbf{x})=\tilde{P}_{m}(\mathbf{x}) / \tilde{P}_{n}(\mathbf{x}) \quad \text { for } 0 \leq m \leq n .
$$


Proof. Comparing (6.5) with (6.2) and (6.3) we get $\phi_{m n}(\mathbf{x})=\tilde{P}_{m}(\mathbf{x}) \phi_{0 n}(\mathbf{x})$. Since $\phi_{m n}(\mathbf{x})$ is positive, so is $\tilde{P}_{m}(\mathbf{x})$ for all $\mathbf{x}$ with $x_{i} \leq 0$. It follows from (6.4) that $\phi_{0 n}(\mathbf{x})=1 / \tilde{P}_{n}(\mathbf{x})$.

LEMMA 6.3. Given $0 \leq m<n$, we can find $c>0$ such that, if $x_{0}^{2}+x_{1}^{2}+\cdots+$ $x_{n-1}^{2}<c^{2}$, then

$$
E \exp \sum_{i=0}^{n-1} \tau_{i}^{m n} x_{i}<\infty .
$$

PROOF. It is enough to show that $E \exp \left(c \sigma^{m n}\right)<\infty$ for some $c>0$. Since the process has the strong Markov property and since jumps are of size +1 or -1 , we have, for any $c>0$,

$$
E \exp \left(c \sigma^{0 n}\right) \geq E \exp \left(c \sigma^{m n}\right),
$$

including the case of infinity. By Keilson's result [4], the distribution of $\sigma^{0 n}$ belongs to $C E_{+}^{n}$. Hence the left-hand side of (6.8) is finite for a small $c>0$. We remark that the proof of the result of Keilson [4] is essentially as follows. For $x \leq 0$, we have, by Lemma 5.2 ,

$$
E \exp \left(x \sigma^{0 n}\right)=\phi_{0 n}(x, x, \ldots)=1 / P_{n}(x),
$$

where $P_{n}(x)=\tilde{P}_{n}(x, x, \ldots)$. The polynomials constitute a system (1.1) satisfying (A.1), (A.2), and $A_{n}>0$. So, by Theorem $2.1, P_{n}(x)$ is a polynomial of degree $n$ having $n$ distinct positive zeros. Hence, by (6.9), the Laplace transform of the distribution of $\sigma^{0 n}$ is that of the convolution of $n$ distinct exponential distributions on $\mathbf{R}_{+}$.

By virtue of Lemma 6.3, we can define $\phi_{m n}(\mathbf{x})$ by (6.1) for $\mathbf{x}$ such that $x_{0}^{2}+$ $\cdots+x_{n-1}^{2}<c^{2}$. Lemmas 6.1 and 6.2 are valid also for this $\mathbf{x}$. To see this, repeat the same proof as before.

It would be worth noting that the system (5.12) of polynomials $Q_{k}^{n}(x)$ is also generalized to the case of many variables. Namely, define

$$
\begin{gathered}
\tilde{Q}_{0}^{n}(\mathbf{x})=1 \text { for } n \geq 0 \\
\tilde{Q}_{1}^{n}(\mathbf{x})=1+\beta_{n-1} / \alpha_{n-1}-x_{n-1} / \alpha_{n-1} \text { for } n \geq 1 \\
\tilde{Q}_{k+1}^{n}(\mathbf{x})=\left(1+\beta_{n-k-1} / \alpha_{n-k-1}-x_{n-k-1} / \alpha_{n-k-1}\right) \tilde{Q}_{k}^{n}(\mathbf{x}) \\
-\left(\beta_{n-k} / \alpha_{n-k}\right) \tilde{Q}_{k-1}^{n}(\mathbf{x}) \text { for } 1 \leq k \leq n-1 .
\end{gathered}
$$

Then the analogues of (5.13) and (5.14) of Lemma 5.4 hold for this case of many variables.

Proof OF Theorem 4.1. We write $f(i)=f_{i}$ and $\mathbf{f}=\left(f_{0}, f_{1}, \ldots\right)$. Define $\phi_{m n}(x)$ and $P_{n}(x)$ by $\phi_{m n}(x \mathbf{f})$ and $\tilde{P}_{n}(x \mathbf{f})$, respectively. Lemma 6.3 shows that the distribution $\mu$ of $T$ has a moment generating function defined in a neighborhood of the origin, which is $\phi_{m n}(x)$ by (4.2). Lemma 6.2 tells that

$$
\phi_{m n}(x)=P_{m}(x) / P_{n}(x) .
$$

In this way the moment generating function of $\mu$ is defined as a rational function on the whole complex plane. The polynomials $P_{n}(x), n=0,1, \ldots$, satisfy $(1.1)$ 
with the constants being

$$
\begin{aligned}
& A_{n}=f_{n} / \alpha_{n} \quad \text { for } n \geq 0, \quad B_{0}=1, \\
& B_{n}=1+\beta_{n} / \alpha_{n}, \quad C_{n}=\beta_{n} / \alpha_{n} \text { for } n \geq 1 .
\end{aligned}
$$

They satisfy (A.1) and (A.2). In fact, we have $P_{n}(0)=1$ for $n \geq 0$. The quantities $N^{+}(n), N^{-}(n)$, and $N(n)$ defined in $\S 2$ coincide with those defined in $\S 4$ for $\mathrm{f}$. Given $0 \leq m<n$, define $k, l, r, s$ as stated in Theorem 4.1. Suppose that $f_{m}>0$ and denote the zeros of $P_{m}(x)$ by $b_{i}^{m}(1 \leq i \leq N(m)=k+r-1)$ in increasing order. Then we have

$$
b_{1}^{m}<\cdots<b_{r}^{m}<0<b_{r+1}^{m}<\cdots<b_{k+r-1}^{m}
$$

by Theorem 2.1. Theorem 2.2 says that we can find zeros $c_{i}(1 \leq i \leq k+r)$ of $P_{n}(x)$ such that

$$
\begin{aligned}
b_{1}^{m} & <c_{1}<b_{2}^{m}<\cdots<c_{r-1}<b_{r}^{m}<c_{r}<0 \\
& <c_{r+1}<b_{r+1}^{m}<c_{r+2}<\cdots<c_{k+r-1}<b_{k+r-1}^{m}<c_{k+r} .
\end{aligned}
$$

The number of the remaining positive [resp. negative] zeros of $P_{n}(x)$ is $l$ [resp. $s$ ] by Theorem 2.1. Using Lemmas 3.1 and 3.2, we see that (4.3) holds in the form of moment generating functions. The case $f_{m}<0$ is treated similarly. Next consider the case $f_{m}=0$. In this case the zeros of $P_{m}(x)$ are $b_{i}^{m}(1 \leq i \leq N(m)=k+r)$ and we have

$$
b_{1}^{m}<\cdots<b_{r}^{m}<0<b_{r+1}^{m}<\cdots<b_{k+r}^{m} .
$$

By Theorem 2.2 there exist zeros $c_{i}(1 \leq i \leq k+r)$ of $P_{n}(x)$ such that

$$
b_{1}^{m}<c_{1}<b_{2}^{m}<\cdots<b_{r}^{m}<c_{r}<0<c_{r+1}<b_{r+1}^{m}<\cdots<c_{k+r}<b_{k+r}^{m} .
$$

There remain $l$ positive zeros and $s$ negative zeros of $P_{n}(x)$. Hence we get (4.5). The proof of Theorem 4.1 is complete. [15].

We remark that, in case $N^{-}(n)=0$, the relation (6.10) is obtained by Wang

PROOF OF THEOREM 4.2. Suppose that the number of common positive [resp. negative] zeros of $P_{m}(x)$ and $P_{n}(x)$ is $p$ [resp. $q$ ]. In the expression (6.10) of $\phi_{m n}(x)$, cancel all factors common in the numerator and the denominator. Letting $b_{i}^{m}(1 \leq i \leq N(m)-p-q)$ denote, in increasing order, the zeros of $P_{m}(x)$ that are not canceled away, we can make the same argument as in the proof of Theorem 4.1. Thus it is proved that (4.3)-(4.5) hold with $k=N^{+}(m+1)-p, l=N^{+}(n)-$ $N(m+1), r=N^{-}(m+1)-q, s=N^{-}(n)-N^{-}(m+1)$. The assertion (iii) is automatic. The number of positive [resp. negative] poles of $\phi_{m n}(x)$ is exactly $k+l$ [resp. $r+s$ ]. If $\mu \in E\left(k^{\prime}, l^{\prime}, r^{\prime}, s^{\prime}\right)$, then the number of positive [resp. negative] poles of $\phi_{m n}(x)$ is at most $k^{\prime}+l^{\prime}$ [resp. $r^{\prime}+s^{\prime}$ ]. Hence the assertion (ii) holds. The assertion (iv) comes from the bounds of $p$ and $q$ in Theorem 2.2. The uniqueness of the pair $k, r$ that satisfies (i) and (ii) is evident. Finally, if $k$ and $r$ satisfy (i) and (iii), then the number of positive [resp. negative] poles of $\phi_{m n}(x)$ is $k+l$ [resp. $r+s$ ], which implies $k$ and $r$ must be $N^{+}(m+1)-p$ and $N^{-}(m+1)-q$, respectively. The proof is complete.

ACKNOWLEDGMENT. The author thanks the referee for suggesting improvement of the Introduction and pointing out an error in the proof of Theorem 2.2 in the manuscript. 


\section{REFERENCES}

1. C. Goldie, A class of infinitely divisible random variables, Proc. Cambridge Philos. Soc. 63 (1967), 1141-1143.

2. I. A. Ibragimov, On the composition of unimodal distributions, Theor. Probab. Appl. 2 (1957), 117-119.

3. S. Karlin and J. L. McGregor, The differential equations of birth-and-death processes and the Stieltjes moment problem, Trans. Amer. Math. Soc. 85 (1957), 489-546.

4. J. Keilson, Log-concavity and log-convexity in passage time densities of diffusion and birth-death processes, J. Appl. Probab. 8 (1971), 391-398.

5. __ On the unimodality of passage time densities in birth-death processes, Statist. Neerlandica 35 (1981), 49-55.

6. J. T. Kent, The appearance of a multivariate exponential distribution in sojourn times for birthdeath and diffusion processes, Probability, Statistics and Analysis, Eds., J. F. C. Kingman et al., London Math. Soc. Lecture Notes Ser., No. 79, 1983, pp. 161-179.

7. W. Ledermann and G. E. H. Reuter, Spectral theory for the differential equations of simple birth and death processes, Philos. Trans. Roy. Soc. London Ser. A 246 (1953-1954), 321-369.

8. U. Rösler, Unimodality of passage times for one-dimensional strong Markov processes, Ann. Probab. 8 (1980), 853-859.

9. K. Sato, Unimodality and bounds of modes for distributions of generalized sojourn times, Stochastic Methods in Biology, Eds., M. Kimura et al., Lecture Notes in Biomath., No. 70, Springer, 1987, pp. 210-221.

10. _ Modes and moments of unimodal distributions, Ann. Statist. Math. 39 (1987), Part A, 407-415.

11. _ Some classes generated by exponential distributions, Probability Theory and Mathematical Statistics, Eds., S. Watanabe et al., Lecture Notes in Math., vol. 1299, Springer, 1988, pp. 454-463.

12. K. Sato and M. Yamazato, On distribution functions of class L, Z. Wahrsch. Verw. Gebiete 43 (1978), 273-308.

13. F. W. Steutel, Note on the infinite divisibililty of exponential mixtures. Ann. Math. Statist. 38 (1967), 1303-1305.

14. __ Preservation of infinite divisibility under mixing and related topics, Math. Centre Tracts 33, Amsterdam, 1970.

15. Wang Zikun, Sojourn times and first passage times for birth and death processes, Sci. Sinica 23 (1980), 269-279.

16. M. Yamazato, Characterization of the class of upward first passage time distributions of birth and death processes and related results, J. Math. Soc. Japan (to appear).

Department of Mathematics, College of General Education, Nagoya UNIVERSITY, NAGOYA, JAPAN 